\title{
Density functional study of dendrimer molecules in solvents of varying quality
}

\author{
Yuchong Zhang, Arjun Valiya Parambathu, and Walter G. Chapman ${ }^{\text {a) }}$ \\ Department of Chemical and Biomolecular Engineering, Rice University, Houston, Texas 77005-1892, USA
}

(Received 15 April 2018; accepted 30 July 2018; published online 14 August 2018)

\begin{abstract}
Modified inhomogeneous statistical associating fluid theory (iSAFT) density functional theory is extended to dendrimer molecules in solvents of varying quality. The detailed structures of isolated dendrimers in implicit solvent are calculated and have a semi-quantitative agreement with simulation results available in the literature. The dendrimers form dense-core structures under all conditions, while their radius of gyration follows different scaling laws. Factors that affect the quality of the solvent are systematically studied in the explicit solvent case. It is found that the solvent size, density, chemical affinity and temperature all play a role in determining a solvent to be good or poor. New molecular dynamics simulations are performed to validate the iSAFT results. Our results provide insight into the phase behavior of dendrimer solutions as well as guidance in practical applications. Published by AIP Publishing. https://doi.org/10.1063/1.5035423
\end{abstract}

\section{INTRODUCTION}

Among all the molecules that feature great potential for applications, dendrimers have received considerable attention. ${ }^{1-3}$ Dendrimers are highly uniform three-dimensional monodisperse polymers with a tree-like globular structure. As illustrated in Fig. 1, a dendrimer molecule consists of three types of monomer segments: branched (B), linear (L), and terminal ( $\mathrm{T}$ ) segments. The branched segments are bonded more than twice, while linear segments are bonded twice and the terminal segments only once. Dendrimers can be characterized by the number of generations $G$, the functionality $b$ of the branched segments, and the spacer between the branched segments $n$. To avoid confusion, we use $G$ as the generation of the dendrimer and $g$ as the generation layer a specific segment belongs to. The total number of monomers in a dendrimer (excluding the core monomer) is given by $N=\frac{n b}{b-2}\left[(b-1)^{G+1}-1\right]$.

Because of their well-defined architecture, dendrimers are applied in dendritic assemblies, layers, films, and liquid crystals. ${ }^{4-7}$ The presence of many terminal groups enables functional modifications to dendrimers, which can be used in medicine, pharmaceuticals, ${ }^{8,9}$ catalysts, chirality, and analytical chemistry. ${ }^{10,11}$ Among the many properties, the knowledge of the detailed structure of dendrimers is fundamental to synthesis as well as application. Specifically, understanding conformations of dendrimers in solvents of varying quality is of great importance. Similar to linear polymers, dendrimers tend to swell in good solvents and collapse in poor solvents. However, dendrimers may have quite different solubility for the same solvent compared with linear polymers, even without any modification of terminal groups. Hawker and Fréchet ${ }^{12}$ found that dendritic polyesters can exhibit a higher solubility

a)Electronic mail:wgchap@ rice.edu than their linear analog. For example, in a solubility test with tetrahydrofuran as the solvent, dendritic polyester was found to have a significantly higher solubility than that of analogous linear polyester. ${ }^{13}$ On the other hand, unlike linear polymers, dendrimers are considered to be able to encapsulate guest molecules ${ }^{14,15}$ and studying their interaction with solvents might shed some light on drug delivery, ${ }^{9,16}$ unimolecular micelles, ${ }^{17,18}$ etc.

Triggered by the unique properties and potential applications, researchers have conducted a large body of experimental studies $^{19-22}$ to explore the relationship between dendrimer and solvents. Theory and simulation methods ${ }^{23-29}$ are also applied and a good summary can be found in the review paper by Ballauff and Likos. ${ }^{30}$ Using molecular dynamics simulations, Murat and $\mathrm{Grest}^{23}$ implicitly incorporated the solvent effect and found that the dendrimers would shrink as the solvent became poorer. They also demonstrated that for all solvent conditions considered, dendrimers obey $R_{g} \sim N^{1 / 3}$ scaling, where $R_{g}$ is the radius of gyration. From Flory theory, Sheng et al. ${ }^{24}$ conclude that the scaling relation should be $R_{g}$ $\sim N^{1 / 5}(G+1)^{2 / 5} n^{2 / 5}$ for a good solvent and $R_{g} \sim N^{1 / 3}$ for a poor solvent, and the latter scaling holds for good solvents only when $n$ is fixed. Based on lattice Monte Carlo and Flory theory, Giupponi and Buzza ${ }^{25}$ also claim that a universal form of $R_{g} \sim N^{1 / 3}$ is incorrect and they propose a scaling $R_{g} \sim N^{1 / 4}(G$ $+1)^{1 / 4} n^{1 / 4}$ for $\theta$ solvent in addition to what Sheng et al. ${ }^{24}$ obtained. Several other studies were conducted with similar or different conclusions. ${ }^{26-29}$ On the other hand, to what extent the dendrimer is affected by varying quality of the solvent remains an active topic for discussion. Several experimental studies ${ }^{19,20}$ found that the solvent effect only plays a role for higher generation dendrimers $(G \geq 4)$ and essentially no collapse occurs for lower generation. Topp et al. ${ }^{21}$ found that even for $G=7$, the poly(amidoamine) (PAMAM) dendrimer showed no apparent sign of collapse from a good solvent (water) to a poor solvent (butanol). On the contrary, using 


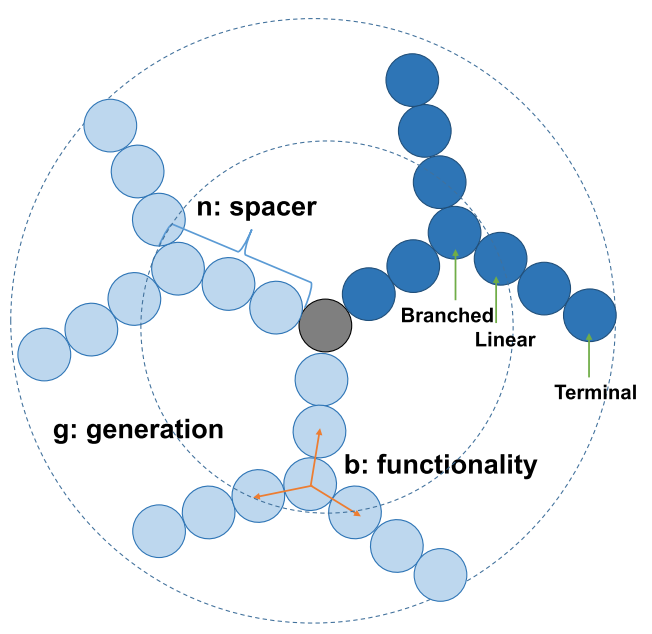

FIG. 1. Schematic of $G 1$ dendrimer with $b=3, n=3$. The monomers within the concentric shell belong to the same generation $g$. The gray bead represents the core segment, and the dark blue group of segments represents one dendron.

multidimensional NMR techniques, Chai et al. ${ }^{22}$ found that even for low generations, poly(propylene imine) (DAB) dendrimers tend to display extended chain conformations in a good solvent (chloroform) and folded chain conformation in a poor solvent (benzene). Also, a number of theoretical or simulation results ${ }^{25,26}$ leads to the conclusion that a significant collapse of the dendrimer takes place for all generations, going from good to poor solvent conditions, although the collapse factor differs case by case. Since there is always ambiguity and controversy over the definition of solvent quality and collapse, plus the differences in the details of the study methods, no definitive conclusions have been drawn in this regard. Recently, Chen et al. ${ }^{31}$ applied classical density functional theory (DFT) to study the conformation of homodendrimers and amphiphilic dendrimers in various solvents and found the existence of dense-core and dense-shell structures under different conditions.

The studies mentioned above are more engaged in predicting how dendrimers of different generations or spacer length behave under certain solvent conditions. However, there is also a large parameter space for the solvent, such as the solvent size, density, and energetic interactions, which upon change would affect the structure and properties of dendrimers. This facet seems to be overlooked by researchers. Our aim for this work is to perform a systematic analysis on how dendrimers are affected by solvents of varying quality and, hopefully, provide some insights on how to construct a good or poor solvent for a specific type of dendrimer.

We use inhomogeneous statistical associating fluid theory $(\text { iSAFT })^{32,33}$ for this task. iSAFT is a density functional theory (DFT) ${ }^{34-37}$ for inhomogeneous complex polymeric fluids. Based on rigorous statistical mechanics, ${ }^{38-41}$ DFT is proved to provide detailed information for fluid structures at a calculation expense advantageous to simulation methods. Following the work of Segura et al. ${ }^{42}$ polyatomic DFTs were developed based on a bulk free energy ${ }^{43,44}$ for associating molecules or a free energy functional. ${ }^{35}$ These DFTs demonstrated that the polyatomic system could be modeled as a mixture of associating atomic fluids in the limit of complete association. A modified DFT is developed to satisfy stoichiometry and got its name as iSAFT by Jain et al. ${ }^{32}$ and is further extended to study branched molecules, ${ }^{45}$ demonstrating the versatility to apply to molecules of different architecture. With appropriate adaptation, iSAFT has been successfully applied to investigate block copolymers in confinement, ${ }^{32}$ tethered polymers, ${ }^{46-49}$ polymer-colloid mixtures, ${ }^{50}$ and micelle formation. ${ }^{51}$ One can also turn to the paper by Emborsky et al. ${ }^{37}$ for a good review.

In this study, we extend iSAFT to study the structures of dendrimer in both implicit and explicit solvents. For implicit cases, there are no actual solvent molecules around but the effect of solvent is incorporated in the interactions between dendrimer segments. By contrast, in explicit cases, the solvent segments are explicitly modeled. Since simulation results available in the literature do not cover the whole range of systems we are interested in, we have also performed molecular dynamics simulations using Large-scale Atomic/Molecular Massively Parallel Simulator (LAMMPS) to justify the iSAFT results, especially for dendrimers in explicit solvents. For clarity, simulation details are included in the Appendix.

The paper is organized as follows. In Sec. II, we explain the general iSAFT model and develop the formalism for a dendrimer centered spherically symmetric system. In Sec. III, we first model the dendrimers in an implicit solvent and compare our results with the simulation results from Murat and Grest ${ }^{23}$ and our own. Several scaling analyses have also been carried out. Then we model dendrimer $G 5$ in an explicit solvent with a discussion of the characteristics of a good or poor solvent and a comparison of our simulation results. Section IV gives our conclusion.

\section{MODEL AND THEORY}

The structure of the dendrimer molecule is already introduced and depicted as Fig. 1. When explicitly incorporated, solvents are modeled as linear chains which may have favorable or unfavorable interactions with the dendrimer. Segments have a temperature-independent diameter $\sigma$, and they interact through pairwise repulsive, attractive, and association contributions, given by the following pair potential:

$$
\begin{aligned}
u\left(\mathbf{r}_{12}, \omega_{1}, \omega_{2}\right)= & u^{\mathrm{ref}}\left(\mathbf{r}_{12}\right)+u^{\mathrm{att}}\left(r_{12}\right) \\
& +\sum_{A} \sum_{B} u_{A B}^{\mathrm{assoc}}\left(\mathbf{r}_{12}, \omega_{1}, \omega_{2}\right),
\end{aligned}
$$

where $r_{12}$ is the distance between two segments and $\omega_{1}, \omega_{2}$ are the orientations of segment 1 and 2 , respectively.

The reference fluid contribution $u^{\text {ref }}$ is often taken as a hard sphere repulsive interaction,

$$
u^{\mathrm{ref}}\left(\mathbf{r}_{12}\right)=u^{\mathrm{hs}}\left(r_{12}\right)=\left\{\begin{array}{l}
\infty, r_{12}<\sigma \\
0, \quad r_{12} \geq \sigma .
\end{array}\right.
$$

The intermolecular attractions $u^{\text {att }}$ are described as a cutshifted Lennard Jones attraction with a Weeks, Chandler, and Andersen (WCA) separation, ${ }^{52,53}$

$$
u^{\mathrm{att}}\left(r_{12}\right)= \begin{cases}u^{\mathrm{LJ}}\left(r_{\min }\right)-u^{\mathrm{LJ}}\left(r_{c}\right) & \text { if } \sigma<r_{12} \leq r_{\min }, \\ u^{\mathrm{LJ}}\left(r_{12}\right)-u^{\mathrm{LJ}}\left(r_{c}\right) & \text { if } r_{\min }<r_{12} \leq r_{c},\end{cases}
$$

where 


$$
u^{\mathrm{LJ}}\left(r_{12}\right)=4 \epsilon^{\mathrm{LJ}}\left[\left(\frac{\sigma}{r_{12}}\right)^{12}-\left(\frac{\sigma}{r_{12}}\right)^{6}\right],
$$

$r_{\min }=2^{1 / 6} \sigma$ is the position of the Lennard Jones potential minimum, and $r_{c}=2.5 \sigma$ is the cutoff distance. The sum of $u^{\mathrm{ref}}$ and $u^{\text {att }}$ serves as a good approximation of the cut-shifted Lennard Jones potential as used in simulation (see the Appendix for details).

Finally, the association potential is given by

$u_{A B}^{\text {assoc }}\left(\mathbf{r}_{12}, \omega_{1}, \omega_{2}\right)= \begin{cases}-\epsilon_{A B}^{a s s o c}, & r_{12}<r_{c} ; \theta_{A 1}<\theta_{c} ; \theta_{B 2}<\theta_{c}, \\ 0, & \text { otherwise },\end{cases}$

where $\theta_{A 1}$ is the angle between the vector from the center of segment 1 to site $\mathrm{A}$ and the vector $\mathbf{r}_{12}$ and $\theta_{B 2}$ is the angle between the vector from the center of segment 2 to site $B$ and the vector $\mathbf{r}_{12}$. We will consider the effect of association in the future work. However, we keep the association contribution in the following derivations to present the general form of the theory.

\section{A. iSAFT density functional theory}

In iSAFT, the polymer is modeled as a flexible chain of tangentially bonded segments. The polymer structure can be linear as well as branched. Traditional DFTs are formulated for an open system in the grand canonical ensemble. So the system is at a fixed volume $(V)$, temperature $(T)$, and chemical potential $(\mu)$ in the presence of an external field $\left(V^{\text {ext }}(\mathbf{r})\right)$. The grand free energy functional $\Omega[\rho]$ for a system of chain fluids can be related to the intrinsic Helmholtz free energy functional $A[\rho]$ as

$$
\Omega\left[\rho_{i}(\mathbf{r})\right]=A\left[\rho_{i}(\mathbf{r})\right]-\sum_{i=1}^{m} \int d \mathbf{r}^{\prime} \rho_{i}\left(\mathbf{r}^{\prime}\right)\left(\mu_{i}-V_{i}^{\mathrm{ext}}\left(\mathbf{r}^{\prime}\right)\right),
$$

where $\rho_{i}$ is the density of segment $i, \mu_{i}$ is its chemical potential, $V_{i}^{\text {ext }}$ is the external field acting on segment $i$, and the sum is over all the $m$ segments of the chain. For the system at equilibrium, the grand free energy is minimized. Minimization of the grand free energy with respect to the density of the segments yields a system of variational equations, known as the Euler-Lagrange equations,

$$
\frac{\delta A\left[\rho_{i}(\mathbf{r})\right]}{\delta \rho_{i}(\mathbf{r})}=\mu_{i}-V_{i}^{\mathrm{ext}}(\mathbf{r}), \forall i=1, \ldots, m .
$$

\section{B. Free energies}

The Helmholtz free energy functional can be decomposed into an ideal and excess contribution,

$$
\begin{aligned}
A\left[\rho_{i}(\mathbf{r})\right]= & A^{\mathrm{id}}\left[\rho_{i}(\mathbf{r})\right]+A^{\mathrm{ex}, \mathrm{hs}}\left[\rho_{i}(\mathbf{r})\right]+A^{\text {ex,chain }}\left[\rho_{i}(\mathbf{r})\right] \\
& +A^{\text {ex,att }}\left[\rho_{i}(\mathbf{r})\right]+A^{\text {ex,assoc }}\left[\rho_{i}(\mathbf{r})\right] .
\end{aligned}
$$

The ideal contribution comes from the ideal gas state of the atomic mixture (id). The excess contribution of the free energy is due to excluded volume effects (hs), chain connectivity (chain), long-range attraction (att), and association (assoc).

The ideal gas functional is known exactly,

$$
\beta A^{\mathrm{id}}\left[\rho_{i}(\mathbf{r})\right]=\int d \mathbf{r}_{1} \sum_{i=1}^{m} \rho_{i}\left(\mathbf{r}_{1}\right)\left[\ln \rho_{i}\left(\mathbf{r}_{1}\right)-1\right] .
$$

The hard sphere contribution $A^{\mathrm{ex}, \mathrm{hs}}\left[\rho_{i}(\mathbf{r})\right]$ is calculated from Rosenfeld's fundamental measure theory $(\mathrm{FMT})^{54}$ for a mixture of hard spheres,

$$
\beta A^{\mathrm{ex}, \mathrm{hs}}\left[\rho_{i}(\mathbf{r})\right]=\int d \mathbf{r}_{1} \Phi\left[n_{\alpha}\left(\mathbf{r}_{1}\right)\right],
$$

where $\Phi\left[n_{\alpha}(\mathbf{r})\right]$ is given by

$$
\begin{aligned}
\Phi\left[n_{\alpha}(\mathbf{r})\right]= & -n_{0} \ln \left(1-n_{3}\right)+\frac{n_{1} n_{2}-\mathbf{n}_{v 1} \cdot \mathbf{n}_{v 2}}{1-n_{3}} \\
& +\frac{n_{2}^{3}-3 n_{2}\left(\mathbf{n}_{v 2} \cdot \mathbf{n}_{v 2}\right)}{24 \pi\left(1-n_{3}\right)^{2}}
\end{aligned}
$$

and $n_{\alpha}(\alpha=0,1,2,3, v 1, v 2)$ are the fundamental measures.

The attraction term $A^{\text {ex,att }}\left[\rho_{i}(\mathbf{r})\right]$ is accounted for by the mean field approximation,, 5

$$
\begin{aligned}
& \beta A^{\mathrm{ex}, \mathrm{att}}\left[\rho_{i}(\mathbf{r})\right] \\
& =\frac{1}{2} \sum_{i=1}^{m} \sum_{j=1}^{m} \int d \mathbf{r}_{1} d \mathbf{r}_{2} \beta u_{i j}^{\mathrm{att}}\left(\left|\mathbf{r}_{2}-\mathbf{r}_{1}\right|\right) \rho_{i}\left(\mathbf{r}_{1}\right) \rho_{j}\left(\mathbf{r}_{2}\right) .
\end{aligned}
$$

Wertheim's first order thermodynamic perturbation theory (TPT1), ${ }^{38-41}$ as extended by Chapman,,${ }^{56}$ is used to calculate $A^{\text {ex,assoc }}\left[\rho_{i}(\mathbf{r})\right]$ and $A^{\text {ex,chain }}\left[\rho_{i}(\mathbf{r})\right]$. The association free energy functional is

$$
\begin{aligned}
\beta A^{\mathrm{ex}, \mathrm{assoc}}\left[\rho_{i}(\mathbf{r})\right]= & \int d \mathbf{r}_{1} \sum_{i=1}^{m} \rho_{i}\left(\mathbf{r}_{1}\right) \\
& \times \sum_{\alpha \in \Gamma^{(i)}}\left(\ln \chi_{\alpha}^{i}\left(\mathbf{r}_{1}\right)-\frac{\chi_{\alpha}^{i}\left(\mathbf{r}_{1}\right)}{2}+\frac{1}{2}\right) .
\end{aligned}
$$

The first summation is over all segments $i$, and the second is over all the association sites on segment $i . \chi_{\alpha}^{i}$ denotes the fraction of segments of type $i$ that are not bonded at their associating site $\alpha$, which can be obtained by the law of mass action, ${ }^{42,56}$

$$
\chi_{\alpha}^{i}\left(\mathbf{r}_{1}\right)=\frac{1}{1+\int d \mathbf{r}_{2} \sum_{j=1}^{m} \rho_{j}\left(\mathbf{r}_{2}\right) \times \sum_{\beta \in \Gamma^{(j)}} \chi_{\beta}^{j}\left(\mathbf{r}_{2}\right) \Delta^{i j}\left(\mathbf{r}_{1}, \mathbf{r}_{2}\right)} .
$$

The association strength $\Delta^{i j}\left(\mathbf{r}_{1}, \mathbf{r}_{2}\right)$ is controlled by

$$
\Delta^{i j}\left(\mathbf{r}_{1}, \mathbf{r}_{2}\right)=K F^{i j}\left(\mathbf{r}_{1}, \mathbf{r}_{2}\right) y^{i j}\left(\mathbf{r}_{1}, \mathbf{r}_{2}\right),
$$

where $K$ is a constant geometric factor which accounts for the entropic cost associated with the orientations and bonding volume of two segments. $F^{i j}\left(\mathbf{r}_{1}, \mathbf{r}_{2}\right)$ is the associating Mayer-f function given as ${ }^{32}$

$$
F^{i j}\left(\mathbf{r}_{1}, \mathbf{r}_{2}\right)=\exp \left[\beta \epsilon_{0}-\beta v_{\text {bond }}^{i j}\left(\mathbf{r}_{1}, \mathbf{r}_{2}\right)\right]-1,
$$

where $\epsilon_{0}$ is the bond energy and $v_{\text {bond }}^{i j}\left(\mathbf{r}_{1}, \mathbf{r}_{2}\right)$ is the bonding potential. In the complete association limit of $\epsilon_{0} \rightarrow \infty$, the chain contribution to the free energy $A^{\text {ex,chain }}\left[\rho_{i}(\mathbf{r})\right]$ can be obtained. For tangentially bonded segments, the bonding potential is given by 


$$
\exp \left[-\beta v_{\text {bond }}^{i j}\left(\mathbf{r}_{1}, \mathbf{r}_{2}\right)\right]=\frac{\delta\left(\left|\mathbf{r}_{1}-\mathbf{r}_{2}\right|-\sigma^{i j}\right)}{4 \pi\left(\sigma^{i j}\right)^{2}}
$$

and $y^{i j}\left(\mathbf{r}_{1}, \mathbf{r}_{2}\right)$ is the cavity correlation function for the inhomogeneous hard sphere reference fluid. $y^{i j}\left(\mathbf{r}_{1}, \mathbf{r}_{2}\right)$ is approximated by $^{32}$

$$
\ln y^{i j}\left(\mathbf{r}_{1}, \mathbf{r}_{2}\right)=\frac{1}{2}\left\{\ln y^{i j}\left[\bar{\rho}_{k}\left(\mathbf{r}_{1}\right)\right]+\ln y^{i j}\left[\bar{\rho}_{k}\left(\mathbf{r}_{2}\right)\right]\right\},
$$

where $\bar{\rho}_{k}\left(\mathbf{r}_{1}\right)$ is the weighted density of segment $k$ at position $\mathbf{r}_{1}$. In the current work, a simple weighting is used,

$$
\bar{\rho}_{k}\left(\mathbf{r}_{1}\right)=\frac{3}{4 \pi\left(\sigma_{k}\right)^{3}} \int_{\left|\mathbf{r}_{1}-\mathbf{r}_{2}\right|<\sigma_{k}} d \mathbf{r}_{2} \rho_{k}\left(\mathbf{r}_{2}\right),
$$

and the expression for $y^{i j}\left[\bar{\rho}_{k}\left(\mathbf{r}_{1}\right)\right]$, the cavity correlation function for the homogeneous hard sphere fluid, is only needed at contact and can be found in the work of Tripathi and Chapman. $^{35}$

\section{Functional derivatives of free energies}

The functional derivatives of the free energies are given as

$$
\begin{gathered}
\frac{\delta \beta A^{\mathrm{id}}\left[\rho_{i}(\mathbf{r})\right]}{\delta \rho_{i}(\mathbf{r})}=\ln \rho_{i}(\mathbf{r}) \\
\frac{\delta \beta A^{\mathrm{ex}, \mathrm{hs}}\left[\rho_{i}(\mathbf{r})\right]}{\delta \rho_{i}(\mathbf{r})}=\int d \mathbf{r}_{1} \frac{\delta \Phi\left[n_{\alpha}\left(\mathbf{r}_{1}\right)\right]}{\delta \rho_{i}(\mathbf{r})} \\
\frac{\delta \beta A^{\mathrm{ex}, \mathrm{att}}\left[\rho_{i}(\mathbf{r})\right]}{\delta \rho_{i}(\mathbf{r})}=\sum_{j=1}^{m} \int_{\left|\mathbf{r}-\mathbf{r}_{1}\right|>\sigma_{i j}} d \mathbf{r}_{1} \beta u_{i j}^{\mathrm{att}}\left(\left|\mathbf{r}-\mathbf{r}_{1}\right|\right) \rho_{j}\left(\mathbf{r}_{1}\right) \\
\frac{\delta \beta A^{\mathrm{ex}, a s s o c}\left[\rho_{i}(\mathbf{r})\right]}{\delta \rho_{i}(\mathbf{r})} \\
=\sum_{\alpha \in \Gamma^{(i)}} \ln \chi_{\alpha}^{i}(\mathbf{r})-\frac{1}{2} \sum_{j=1}^{m} \sum_{k=1}^{m} \int d \mathbf{r}_{1} d \mathbf{r}_{2} \rho_{j}\left(\mathbf{r}_{1}\right) \rho_{k}\left(\mathbf{r}_{2}\right) \\
\times \sum_{\left.\alpha \in \Gamma^{(j)}\right)} \sum_{\beta \in \Gamma^{(k)}} \chi_{\alpha}^{j}\left(\mathbf{r}_{1}\right) \chi_{\beta}^{k}\left(\mathbf{r}_{2}\right) \frac{\delta \Delta^{j k}\left(\mathbf{r}_{1}, \mathbf{r}_{2}\right)}{\delta \rho_{i}(\mathbf{r})}
\end{gathered}
$$

The functional derivative of the association free energy is derived by Bymaster and Chapman ${ }^{33}$ and applies to the full range of association. Further applying the limit of complete association gives

$$
\begin{aligned}
\frac{\delta \beta A^{\text {ex,chain }}\left[\rho_{i}(\mathbf{r})\right]}{\delta \rho_{i}(\mathbf{r})}= & \sum_{\alpha \in \Gamma^{(i)}} \ln \chi_{\alpha}^{i}(\mathbf{r})-\frac{1}{2} \sum_{j=1}^{m} \sum_{j^{\prime}}^{\left\{j^{\prime}\right\}} \int d \mathbf{r}_{1} \rho_{j}\left(\mathbf{r}_{1}\right) \\
& \times \frac{\delta \ln y^{j j^{\prime}}\left[\bar{\rho}_{k}\left(\mathbf{r}_{1}\right)\right]}{\delta \rho_{i}(\mathbf{r})}
\end{aligned}
$$

where $\left\{j^{\prime}\right\}$ is the set of all segments bonded to segment $j$. In this equation, the first term on the right side enforces stoichiometry. The cavity correlation function is further approximated by its bulk counterpart evaluated at the weighted density as Eq. (18).

Substituting the functional derivatives of the free energies in the Euler-Lagrange equation Eq. (7) gives

$$
\ln \rho_{i}(\mathbf{r})+\sum_{\alpha \in \Gamma^{(i)}} \ln \chi_{\alpha}^{i}(\mathbf{r})=D_{i}(\mathbf{r})+\beta\left(\mu_{i}-V_{i}^{\mathrm{ext}}(\mathbf{r})\right),
$$

where $D_{i}(\mathbf{r})$ is given by

$$
\begin{aligned}
D_{i}(\mathbf{r})= & -\frac{\delta \beta A^{\mathrm{ex}, \mathrm{hs}}\left[\rho_{i}(\mathbf{r})\right]}{\delta \rho_{i}(\mathbf{r})}-\frac{\delta \beta A^{\mathrm{ex}, \mathrm{att}}\left[\rho_{i}(\mathbf{r})\right]}{\delta \rho_{i}(\mathbf{r})} \\
& -\frac{\delta \beta A^{\mathrm{ex}, \mathrm{assoc}}\left[\rho_{i}(\mathbf{r})\right]}{\delta \rho_{i}(\mathbf{r})}+\frac{1}{2} \sum_{j=1}^{m} \sum_{j^{\prime}}^{\left\{j^{\prime}\right\}} \int d \mathbf{r}_{1} \rho_{j}\left(\mathbf{r}_{1}\right) \\
& \times \frac{\delta \ln y^{j j^{\prime}}\left[\bar{\rho}_{k}\left(\mathbf{r}_{1}\right)\right]}{\delta \rho_{i}(\mathbf{r})} .
\end{aligned}
$$

Note that $\chi_{\alpha}^{i}(\mathbf{r})$ in Eq. (25) only refer to sites contributing to chain formation, not association.

\section{Equilibrium density profile for a dendrimer centered spherical system}

Solving Eq. (25) in three dimensions is challenging because the density distribution can vary in every dimension. However, in the case of an isolated dendrimer with the center core fixed, due to the spherical symmetry of the dendrimer structure, it is possible to simplify a $3 \mathrm{D}$ problem to a $1 \mathrm{D}$ problem. In such a model, all segment densities are only functions of the radial distance from the center core and we are able to focus on a single dendron of the dendrimer, which is the branch formed at the initial branching point (see Fig. 1). Supposing the center core has a diameter of $\sigma_{C}$, and all the other monomer or solvent segments have a diameter of $\sigma_{D}$. We denote the segment that is directly tethered to the center core as segment 1 , and the subsequent ones $2,3, \ldots, m_{D}$. To be noted is that $m_{D}$ is not the number of total segments in the dendron but the number of distinguishable segments, which is equal to the path length from segment 1 to a terminal segment, as illustrated in Fig. 2.

It is easy to obtain that $m_{D}=(G+1) \times n$. Segment $i$ has a total number of $2^{g}$ equivalents in one dendron, for $g n<i$ $\leq(g+1) n$. The external field exerted by the center core on the monomer segment 1 is

$$
V_{1}^{\mathrm{ext}}(r)= \begin{cases}u & \text { if } r=\sigma_{0} \\ \infty & \text { otherwise }\end{cases}
$$

where $\sigma_{0}=\frac{\sigma_{C}+\sigma_{D}}{2}$ and $u$ sets the grafting density or number of arms on the center core segment.

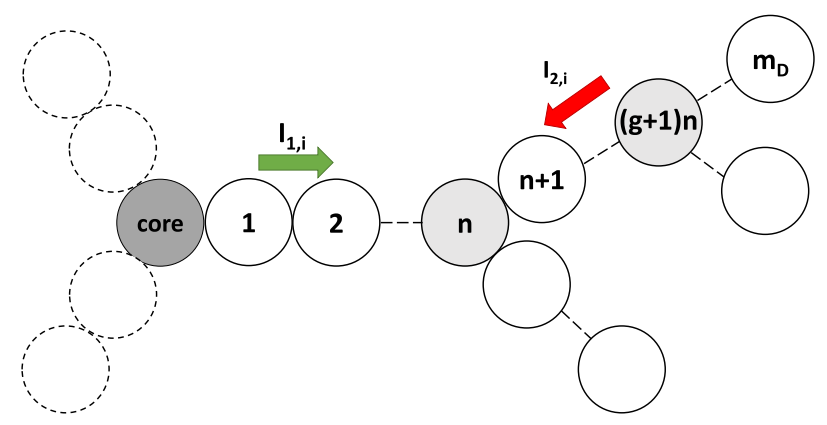

FIG. 2. Schematic of the way monomer segments are indexed in a dendron of dendrimer. Light gray bead indicates branched segment and the dark gray bead is the core segment. Dashed beads represent segments of other dendrons. $I_{1, i}$ and $I_{2, i}$ are pointing outwards and inwards, respectively. 
For the other monomer or solvent segments,

$$
V_{i}^{\text {ext }}(r)= \begin{cases}\infty & \text { if } r<\sigma_{0} \\ 0 & \text { otherwise }\end{cases}
$$

The density of dendrimer segments can be expressed as follows. For segment 1 ,

$$
\rho_{1}\left(r_{1}\right)= \begin{cases}\exp \left(\beta \mu_{D}-\beta u\right) \exp \left[D_{1}\left(\sigma_{0}\right)\right] I_{1,1}\left(\sigma_{0}\right) I_{2,1}\left(\sigma_{0}\right) \delta\left(r_{1}-\sigma_{0}\right) & \text { if } 1 \text { is a } \mathrm{L} \text { or T segment } \\ \exp \left(\beta \mu_{D}-\beta u\right) \exp \left[D_{1}\left(\sigma_{0}\right)\right] I_{1,1}\left(\sigma_{0}\right) I_{2,1}^{b-1}\left(\sigma_{0}\right) \delta\left(r_{1}-\sigma_{0}\right) & \text { if } 1 \text { is a B segment. }\end{cases}
$$

The delta function $\delta\left(r_{1}-\sigma_{0}\right)$ accounts for the fact that segment 1 is tethered at a fixed distance $\left(\sigma_{0}\right)$ from origin, and for the other monomer segments $i=2, \ldots, m_{D}$,

$$
\rho_{i}\left(r_{i}\right)= \begin{cases}\exp \left(\beta \mu_{D}-\beta u\right) \exp \left[D_{i}\left(r_{i}\right)\right] I_{1, i}\left(r_{i}\right) I_{2, i}\left(r_{i}\right) & \text { if } i \text { is a } \mathrm{L} \text { or T segment } \\ \exp \left(\beta \mu_{D}-\beta u\right) \exp \left[D_{i}\left(r_{i}\right)\right] I_{1, i}\left(r_{i}\right) I_{2, i}^{b-1}\left(r_{i}\right) & \text { if } i \text { is a B segment, }\end{cases}
$$

where $\mu_{D}$ is the "chemical potential" of the dendron imposed by the tether condition. $I_{1, i}$ and $I_{2, i}$ are multiple integrals introduced by Jain et al. ${ }^{32}$ to facilitate solving the Euler-Lagrange equations for linear chains and further extended to branched chains. ${ }^{45}$ The integrals are solved using the following recurrence:

$$
\begin{gathered}
I_{1,1}\left(\sigma_{0}\right)=1, \\
I_{1,2}\left(r_{2}\right)= \begin{cases}\exp \left[D_{1}\left(\sigma_{0}\right)\right] \Delta^{(1,2)}\left(\sigma_{0}, r_{2}\right) & \text { if } 1 \text { is a } \mathrm{L} \text { or T segment, } \\
I_{2,1}^{b-2}\left(\sigma_{0}\right) \exp \left[D_{1}\left(\sigma_{0}\right)\right] \Delta^{(1,2)}\left(\sigma_{0}, r_{2}\right) & \text { if } 1 \text { is a B segment, }\end{cases} \\
I_{1, i}\left(r_{i}\right)= \begin{cases}\int I_{1, i-1}\left(r_{i-1}\right) \exp \left[D_{i-1}\left(r_{i-1}\right)\right] \Delta^{(i-1, i)}\left(r_{i-1}, r_{i}\right) d r_{i-1} & \text { if } i-1 \text { is a L or T segment, } \\
\int I_{1, i-1}\left(r_{i-1}\right) I_{2, i-1}^{b-2}\left(r_{i-1}\right) \exp \left[D_{i-1}\left(r_{i-1}\right)\right] \Delta^{(i-1, i)}\left(r_{i-1}, r_{i}\right) d r_{i-1} & \text { if } i \text { is a B segment, }\end{cases}
\end{gathered}
$$

and

$$
\begin{gathered}
I_{2, m_{D}}\left(r_{m_{D}}\right)=1, \\
I_{2, i}\left(r_{i}\right)= \begin{cases}\int I_{2, i+1}\left(r_{i+1}\right) \exp \left[D_{i+1}\left(r_{i+1}\right)\right] \Delta^{(i, i+1)}\left(r_{i}, r_{i+1}\right) d r_{i+1} & \text { if } i+1 \text { is a } \mathrm{L} \text { or T segment, } \\
\int I_{2, i+1}^{b-1}\left(r_{i+1}\right) \exp \left[D_{i+1}\left(r_{i+1}\right)\right] \Delta^{(i, i+1)}\left(r_{i}, r_{i+1}\right) d r_{i+1} & \text { if } i+1 \text { is a B segment, }\end{cases} \\
I_{2,1}\left(\sigma_{0}\right)= \begin{cases}\int I_{2,2}\left(r_{2}\right) \exp \left[D_{2}\left(r_{2}\right)\right] \Delta^{(1,2)}\left(\sigma_{0}, r_{2}\right) d r_{2} & \text { if } 2 \text { is a L or T segment, } \\
\int I_{2,2}^{b-1}\left(r_{2}\right) \exp \left[D_{2}\left(r_{2}\right)\right] \Delta^{(1,2)}\left(\sigma_{0}, r_{2}\right) d r_{2} & \text { if } 2 \text { is a B segment. }\end{cases}
\end{gathered}
$$

$I_{1, i}$ and $I_{2, i}$ can be considered as integration operators propagating outwards and inwards, respectively, as illustrated by Fig. 2 . In this way, the chemical potential of a segment in a dendron depends on the local chemical potential of all other segments in the dendron. The total density of dendrimer segments is calculated as

$$
\rho_{D}(r)=\sum_{i=1}^{m_{D}} 2^{g} \rho_{i}(r) \quad \text { for } r>\sigma_{0}
$$

The value of $\exp \left(\beta \mu_{D}-\beta u\right)$ can be obtained from the grafting density $\rho_{g}$, which is the number of segments 1 per unit area of the excluding surface:

$$
\int \rho_{1}(r) d r=\rho_{g}=\frac{b}{4 \pi \sigma_{0}^{2}}
$$

Then we have

$$
\exp \left(\beta \mu_{D}-\beta u\right)= \begin{cases}\frac{\rho_{g}}{\exp \left[D_{1}\left(\sigma_{0}\right)\right] I_{1,1}\left(\sigma_{0}\right) I_{2,1}\left(\sigma_{0}\right)} & \text { if } 1 \text { is a } \mathrm{L} \text { or T segment } \\ \frac{\rho_{g}}{\exp \left[D_{1}\left(\sigma_{0}\right)\right] I_{1,1}\left(\sigma_{0}\right) I_{2,1}^{b-1}\left(\sigma_{0}\right)} & \text { if } 1 \text { is a B segment. }\end{cases}
$$

Substitute the equation into Eq. (30) gives the density profile of all the other monomer segments.

The density profile of solvent molecules is more straightforward to obtain since the segments are free to move for $r>\sigma_{0}$. For a solvent consisting of $m_{S}$ segments, we have

$$
\rho_{j}\left(r_{j}\right)=\exp \left(\beta \mu_{S}\right) \exp \left[D_{j}\left(r_{j}\right)\right] I_{1, j}\left(r_{j}\right) I_{2, j}\left(r_{j}\right) .
$$

Here $\mu_{S}$ is the chemical potential of the solvent in the bulk and $I_{1, j}, I_{2, j}$ are the following:

$$
I_{1,1}\left(r_{1}\right)=1
$$


$I_{1, j}\left(r_{j}\right)=\int I_{1, j-1}\left(r_{j-1}\right) \exp \left[D_{j-1}\left(r_{j-1}\right)\right] \Delta^{(j-1, j)}\left(r_{j-1}, r_{j}\right) d r_{j-1}$,

and

$$
\begin{gathered}
I_{2, m_{S}}\left(r_{m_{S}}\right)=1 \\
I_{2, j}\left(r_{j}\right)=\int I_{2, j+1}\left(r_{j+1}\right) \exp \left[D_{j+1}\left(r_{j+1}\right)\right] \Delta^{(j+1, j)}\left(r_{j+1}, r_{j}\right) d r_{j+1} .
\end{gathered}
$$

The total density of solvent segments is calculated as

$$
\rho_{S}(r)=\sum_{j=1}^{m_{S}} \rho_{j}(r) \quad \text { for } r>\sigma_{0} .
$$

For most cases studied, we follow the type of dendrimer as constructed in the simulation work of Murat and Grest, ${ }^{23}$ with $b=3$ and $n=7$. The spacer number $n$ is varied for a few cases where we work on scaling. Dendrimers up to $G 8$ are modeled using iSAFT. In this work, we consider the sizes of all dendrimer and solvent segments to be the same, such that $\sigma_{C}=\sigma_{D}=\sigma_{0}=\sigma$. Picard's iteration method is applied to solve the set of Eq. (30) to find the equilibrium density profile.

\section{RESULTS AND DISCUSSION}

\section{A. Dendrimer in implicit solvent}

We first investigate the case where an isolated dendrimer is immersed in an implicit solvent. The simulation results of Murat and Grest ${ }^{23}$ show that in the limit of an implicit athermal solvent (equivalently, temperature being infinity and nonbonded interaction reducing to pure hard sphere repulsion), dendrimer segments exhibit pure repulsion and the solvent is considered a good solvent since the dendrimer is swollen. As the reduced temperature, $T^{*}=k_{b} T / \epsilon^{\mathrm{LJ}}$ goes down, the solvent becomes poor and the dendrimer tends to collapse.

With iSAFT we are able to model the structure of dendrimer for the same conditions and make a direct comparison with the simulation. Figure 3 shows radial monomer densities in the implicit athermal solvent from $G 5$ to $G 8$ with the densities offset by 0.1 for clarity. Overall, the theoretical results are in semi-quantitative agreement with the simulation data.

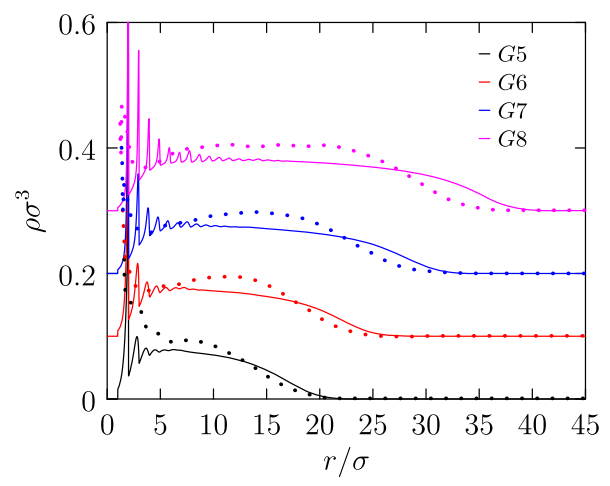

FIG. 3. Radial monomer densities of dendrimers in an implicit athermal solvent. The solid lines represent iSAFT results and the dotted lines are the simulation results of Murat and Grest. ${ }^{23}$ Results for $G 6, G 7$, and $G 8$ are shifted up by $0.1,0.2$, and 0.3 , respectively.
Similar to simulation, the density from iSAFT reaches a local minimum before $r=5 \sigma$ though there is strong oscillation near the origin as a result of the packing effect, which is characteristic of DFT methods. The density profile continues to grow and arrives at a region of an almost constant density before it drops monotonically and vanishes. Simulation appears to show a slight density maximum where theory shows more even distribution of monomers. While the plateau is more apparent for higher generations from both methods, it is interesting to find that the heights of plateau for all generations are almost the same $\left(\rho \sigma^{3} \approx 0.1\right.$ from simulation and $\rho \sigma^{3} \approx 0.08$ from iSAFT).

Concerning the differences between the theoretical and simulation results, it would be instructive to look into the individual density distributions of monomer segments belonging to various generations. To obtain generation by generation density profiles from simulation, we have implemented our LAMMPS code for this system. As we see from Fig. 4, for inner generations (small $g$ ), a peak structure is typical, which indicates a relatively localized distribution. However, this kind of localization is not as apparent as that from simulation. From iSAFT, the density profile of every generation rises quickly near the origin and as a result, patterns of the plateau structure appear as early as $g 2$. On the other hand, in simulation, an additional shoulder is formed starting from $g 5$. This may be accounted by the fact that in iSAFT, although there is penalty associated with dense packing, there is no constraint on the bond angle between segments. This also explains why the overall density profile from iSAFT is slightly flatter than simulation. However, it is clear to see that monomer segments belonging to the outer generations cover the whole range almost uniformly, which is the same case in simulation and confirmed by other studies. ${ }^{25,27,29}$ The folding back of outer generations indicate that although being compact, the dendrimer is quite flexible and open for penetration. We also make a comparison for the radial monomer densities from $G 5$ to $G 8$ at $T^{*}=3$. Again, we find semi-quantitative agreement between theory and simulation, as shown in Fig. 5. Under this condition, the plateau of the density profile from iSAFT is lower than that from simulation $\left(\rho \sigma^{3} \approx 0.3\right.$ from iSAFT and $\rho \sigma^{3} \approx 0.35$ from simulation) and wider as well. Individual density distributions of monomer segments belonging to various generations are depicted in Fig. 6. The break-down structure is similar to that

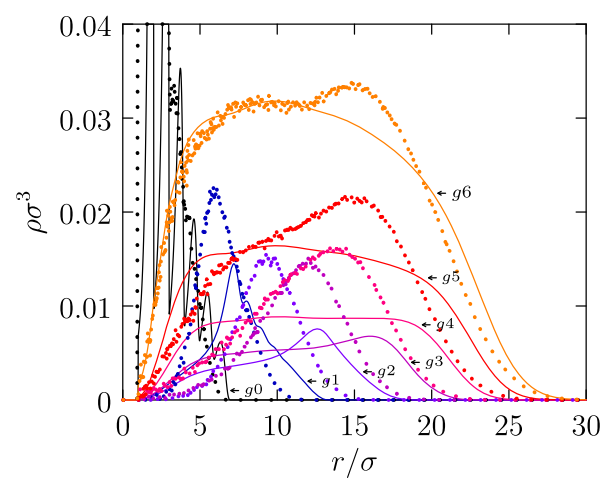

FIG. 4. Contributions to the overall monomer density profile for $G 6$ dendrimer in an implicit athermal solvent, due to monomers of generation $g$. Solid lines represent iSAFT results and dotted lines are our MD results. 


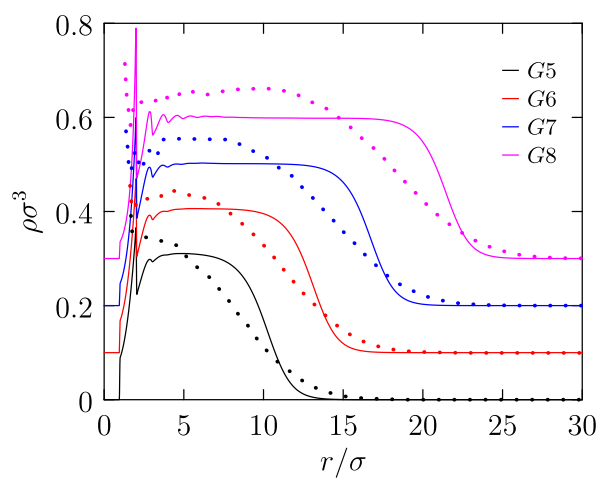

FIG. 5. Radial monomer densities of dendrimers at $T^{*}=3$. The solid lines represent iSAFT results and the dotted lines are the simulation results of Murat and Grest. ${ }^{23}$ Results for $G 6, G 7$ and $G 8$ are shifted up by $0.1,0.2$ and 0.3 , respectively.

under athermal conditions except that density profile of each generation is compressed and raised, corresponding to a partially collapsed structure. It should be mentioned that from simulation, $T^{*}=3$ is identified as the $\theta$ temperature (at which the dendrimer acts just like an ideal chain and the corresponding solvent is defined as the $\theta$ solvent), while from iSAFT, it is more like a poor solvent condition. That is, the $\theta$ temperature from iSAFT tends to be higher. It is also interesting to see how a dendrimer of specific generation behaves differently in a solvent of varying quality. In Fig. 7, we plot the monomer densities of $G 8$ for the athermal condition, $T^{*}=4$ and $T^{*}=3$, respectively. As the quality of the solvent becomes poorer, the dendrimer transitions from a swollen structure to a collapsed one. This is because the monomer segments prefer to aggregate to reduce contact with the solvents when the dendrimer-solvent interaction is unfavorable. With the monomer density profile, we are able to calculate the radius of gyration of dendrimer $R_{g}$ from

$$
R_{g}^{2}=\frac{\int \rho_{D}(r) r^{4} d r}{\int \rho_{D}(r) r^{2} d r}
$$
Table I.

Results of $R_{g}$ at different conditions are listed in

To implement some simple scaling analysis, we first fix the spacer number $n$ and vary the generation number $G$. As shown in Fig. 8, relationships of $R_{g}$ versus $N$ under different conditions are plotted in a double-logarithmic scale. Dendrimers

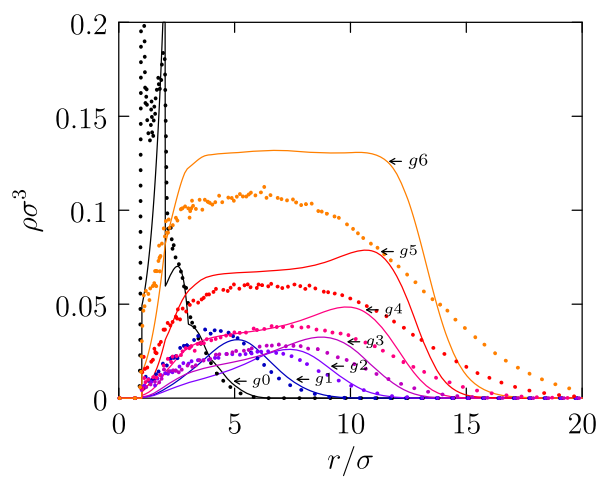

FIG. 6. Contributions to the overall monomer density profile for $G 6$ dendrimer at $T^{*}=3$, due to monomers of generation $g$. Solid lines represent iSAFT results and dotted lines are our MD results.

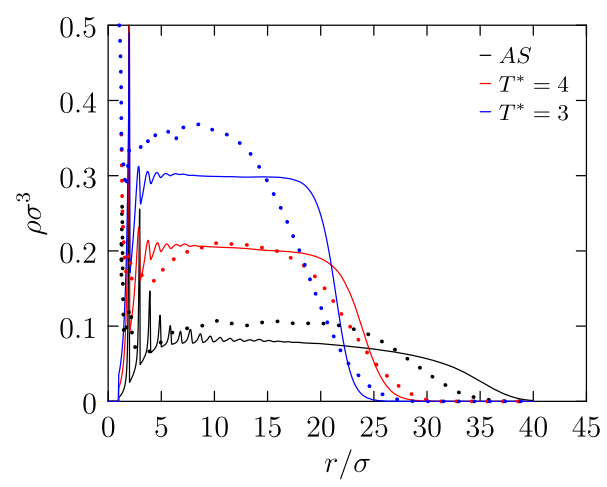

FIG. 7. Radial monomer densities of $G 8$ dendrimers in solvents of varying quality. The solid lines represent iSAFT results and the dotted lines come from the simulation results of Murat and Grest. ${ }^{23}$

of $G 5$ through $G 8$ are presented and for the athermal condition, we include $G 1$ to $G 4$ as well. Similar to the results of Murat and Grest,$^{23}$ for the athermal case, a linear relationship is not formed until some large $G$. Following their way, only points of $G 5$ to $G 8$ are fit to the form $R_{g}=A N^{v}$. The value of $v$ is 0.32 for the athermal case and for $T^{*}=2,0.33$ for $T^{*}=3$. Inverting the relation, we find $N \sim R_{g}^{3}$ for all the cases, which is also consistent with the conclusion of Murat and Grest.

However, as pointed out by several studies, ${ }^{24-29}$ the scaling law for dendrimers should behave in a more complicated way, and the form of $R_{g} \sim N^{1 / 3}$ cannot apply to dendrimers at all conditions. A good way of testing is to repeat the above analysis with a fixed generation number $G$ and a varied spacer number $n$. $G$ is fixed at 5 in our analysis and the new scaling relation is plotted in Fig. 9. As the exact value of $\theta$ temperature is not determined, we only plot the athermal and $T^{*}=2$ cases, which should well represent the good and poor solvent cases,

TABLE I. Radius of gyration of dendrimers.

\begin{tabular}{lrrrr}
\hline \hline$G$ & $N$ & $n$ & $T$ & \multicolumn{1}{c}{$R_{g}^{2}$} \\
\hline 8 & 10731 & 7 & a.s. & 707.7 \\
8 & 10731 & 7 & 3.0 & 280.3 \\
8 & 10731 & 7 & 2.0 & 202.0 \\
7 & 5355 & 7 & a.s. & 463.8 \\
7 & 5355 & 7 & 3.0 & 173.4 \\
7 & 5355 & 7 & 2.0 & 128.8 \\
6 & 2667 & 7 & a.s. & 296.3 \\
6 & 2667 & 7 & 3.0 & 109.0 \\
6 & 2667 & 7 & 2.0 & 81.1 \\
5 & 1323 & 7 & a.s. & 183.8 \\
5 & 1323 & 7 & 3.0 & 69.6 \\
5 & 1323 & 7 & 2.0 & 52.1 \\
5 & 945 & 5 & a.s. & 123.9 \\
5 & 945 & 5 & 3.0 & 54.8 \\
5 & 945 & 5 & 2.0 & 41.3 \\
5 & 567 & 3 & a.s. & 65.3 \\
5 & 567 & 3 & 3.0 & 38.2 \\
5 & 567 & 3 & 2.0 & 29.9 \\
4 & 651 & 7 & a.s. & 100.4 \\
3 & 315 & 7 & a.s. & 64.5 \\
2 & 147 & 7 & a.s. & 36.0 \\
1 & 63 & 7 & a.s. & 18.1 \\
\hline \hline
\end{tabular}




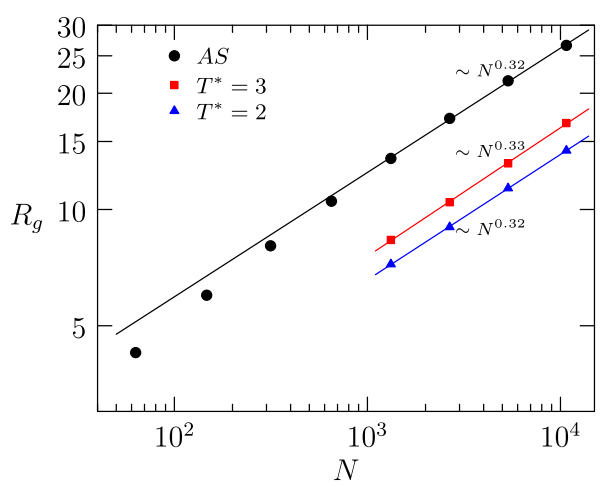

FIG. 8. Radius of gyration of the dendrimers as a function of the number of monomers in the dendrimer when spacer number $n$ is fixed at 7, on a doublelogarithmic scale. The points correspond to the calculated results from iSAFT, and the full lines are best fits to the form $R_{g}=A N^{v}$ for $5 \leq G \leq 8$.

respectively. The fitting line shows that $v=0.61$ for the athermal case and $v=0.33$ for $T^{*}=2$. We also notice that at fixed $G$, the scaling law for a good solvent, $R_{g} \sim N^{1 / 5}(G+1)^{2 / 5} n^{2 / 5}$ by Sheng et al. ${ }^{24}$ reduces to $R_{g} \sim N^{3 / 5}$ and the scaling law for a poor solvent stays as $R_{g} \sim N^{1 / 3}$. The scaling exponents for both cases agree well and we believe that the iSAFT theory will provide meaningful and reasonable scaling of dendrimers. A more complete and systematic study is required to validate any scaling law. For example, dendrimers of a wider range of $G$ should be tested when varying $n$. However, that is not the focus of this work.

\section{B. Dendrimer in explicit solvent}

While the implicit solvent effect is successfully captured and well explained, it is a more realistic model when solvent exists explicitly. In iSAFT, explicit solvent is readily introduced into the system and unlike simulation methods, only little calculation expense is added to the task. By tuning parameters in the model, we want to understand how both internal (size, density, and chemical affinity) and external (temperature) factors have an impact on the solvent quality. Both low and high reduced solvent densities are considered. While the high liquid-like density system is quite common for dendrimer solutions, the low gas-like solvent system can model gas adsorption on dendrimers and provide insights on applications such as chromatography, ${ }^{57}$ sensor, ${ }^{58}$ and gas separation. ${ }^{59}$ In addition, we learn from the implicit solvent case

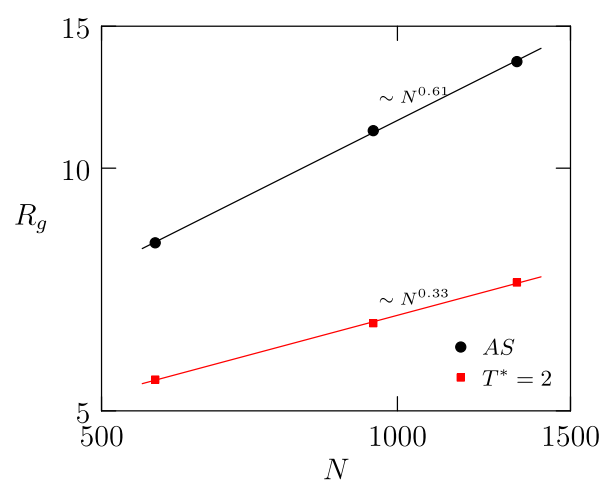

FIG. 9. Radius of gyration of the dendrimers as a function of the number of monomers in the dendrimer when generation number $G$ is fixed at 5 , on a double-logarithmic scale. The points correspond to the calculated results from iSAFT, and the full lines are best fits to the form $R_{g}=A N^{v}$.

that dendrimers of different generations behave similarly at a given solvent condition. Therefore, the upcoming results and discussion are based on 65 dendrimers. For some systems, simulation results are provided from our molecular dynamics code.

We start with a dendrimer made up of hard sphere segments immersed in a hard chain solvent. At this athermal condition, all segments exhibit pure repulsion toward each other. Figures 10(a) and 10(b) show the distribution of monomer and solvent segments at the fixed bulk density of solvent segments of 0.1 and 0.6 , respectively. The chain length of solvent $N_{S}$ ranges from 1 to 100 with the bond length equal to $\sigma$. The density profile of an athermal dendrimer in an implicit solvent is plotted as well, and it has a more extended structure than any of the explicit cases. The explicit solvent freely penetrates inside the dendrimer. At low solvent density [Fig. 10(a)], we notice that as the solvent chain grows longer, it is more difficult for solvent segments to migrate inside the dendrimer and the dendrimer tends to be more compressed. This is owing to the increased excluded volume of longer chains. A similar trend is observed at a high density [Fig. 10(b)], while we see a more facilitated penetration for long chains, which may be accounted by a greater chemical potential exerted by the solvent at a higher bulk density. For both densities, in the case of a spherical solvent $\left(N_{S}=1\right)$, the solvent density gradually declines from the bulk density when entering the dendrimer and stays almost constant till near the origin. It is more clearly

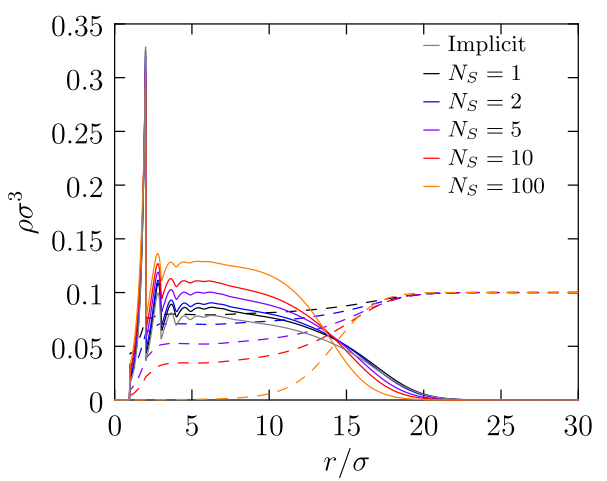

(a)

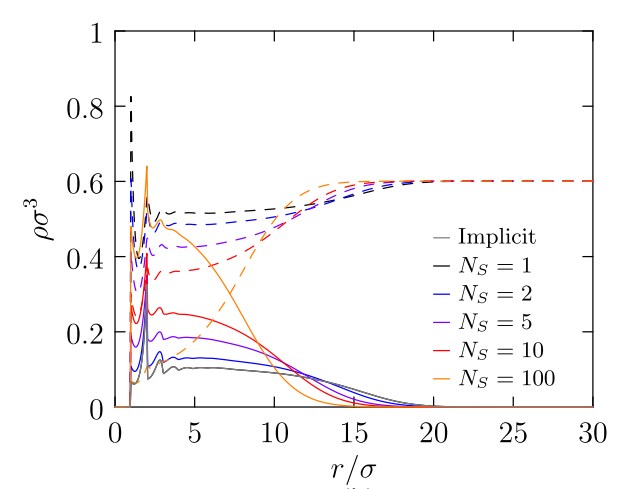

(b)
FIG. 10. Density profiles of $G 5$ dendrimer in solvents of different chain length. Solid curves represent dendrimer monomers, and dashed curves represent solvent segments. For reference, the dendrimer density profile in implicit athermal solvent is also plotted. (a) $\rho_{S} \sigma^{3}=0.1$ at bulk. (b) $\rho_{S} \sigma^{3}=0.6$ at bulk. 


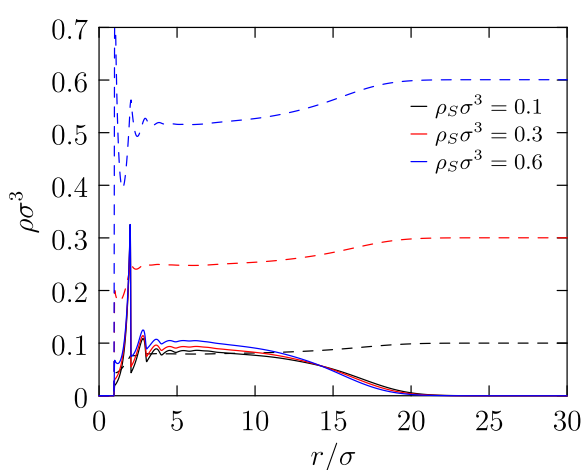

(a)

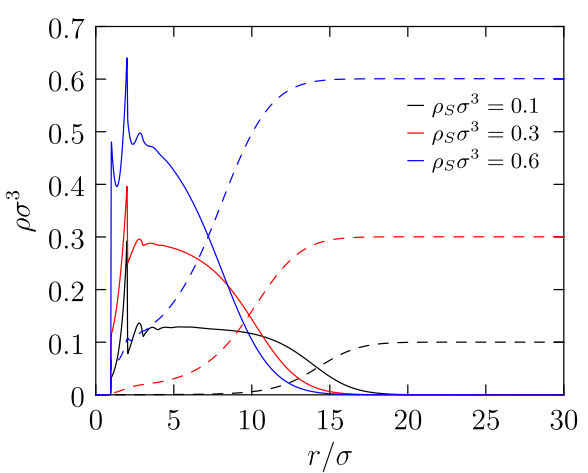

(b)
FIG. 11. Density profiles of G5 dendrimer in solvents at different bulk solvent densities. Solid curves represent dendrimer monomers, and dashed curves represent solvent segments. (a) $N_{S}=1$. (b) $N_{S}=100$. illustrated by Fig. 11(a). Here the solvent length is fixed as 1 , while the bulk density is varied. The solvent density profiles show similar trends, and the dendrimer structure is barely affected for any bulk density. On the contrary, in Fig. 11(b), where the solvent length is 100 , the solvent density profile takes a sharp drop and the dendrimer structure is very sensitive to the bulk density of solvent.

Now we introduce the attraction between dendrimer and solvent segments. $\epsilon_{D D}^{L J}, \epsilon_{S S}^{L J}$, and $\epsilon_{D S}^{L J}$ are the attraction energy between dendrimer segments, solvent segments and, dendrimer and solvent segments, respectively. We assume $\epsilon_{D D}^{L J}$ $=\epsilon_{S S}^{L J}$ and a spherical solvent for all cases studied. $\epsilon_{D S}^{L J}$ is an indicator of the affinity between dendrimer and solvent segments. When $\epsilon_{D S}^{L J}=0$, there is only pure repulsion between the dendrimer and solvent. When $\epsilon_{D S}^{L J}=\epsilon_{D D}^{L J}$, there is no difference between a dendrimer segment and a solvent segment energetically. Figures 12(a) and 12(b) show from simulation and theory how the structure of dendrimer varies for an explicit solvent of different $\beta \epsilon_{D S}^{L J}$ with $\beta \epsilon_{D D}^{L J}$ fixed as 1 . As $\beta \epsilon_{D S}^{L J}$ increases, we see a growing influx of solvent into the dendrimer and there is even some enhancement over the bulk density at the dendrimer periphery. However, it is a bit surprising that at low density [Fig. 12(a)], the dendrimer still retains a relatively compressed conformation at $\beta \epsilon_{D S}^{L J}=1$. On the contrary, at high density [Fig. 12(b)], a well swollen structure is observed at $\beta \epsilon_{D S}^{L J}=1$. This finding seems to contradict what we have from Figs. 11(a) and 11(b) that the dendrimer tends to be more extended under a lower bulk solvent density when all the other conditions are the same. A proper explanation is that the fluid structure is the result of the interplay between all segments. The attraction between dendrimer segments makes the dendrimer compact at low solvent density and the dendrimer likes to expand to avoid extremely dense packing inside the dendrimer when the solvent is abundant. The agreement between iSAFT and simulation results is good except for the case of $\beta \epsilon_{D D}^{L J}=1, \beta \epsilon_{D S}^{L J}=1$ at $\rho_{S} \sigma^{3}=0.1$ [blue curve in Fig. 12(a)]. The simulation results show a slightly more extended structure of the dendrimer and much more enhanced absorption of solvents inside the dendrimer. Again, this is explained by the lack of intramolecular correlation in the iSAFT theory.

The different fluid structures at $\beta \epsilon_{D D}^{L J}=\beta \epsilon_{D S}^{L J}=0$ and $\beta \epsilon_{D D}^{L J}=\beta \epsilon_{D S}^{L J}=1$ [compare Figs. 11(a) and 12] can also be interpreted as a result of varying temperature for a specific dendrimer and solvent combination. To better understanding the temperature effect, we plot dendrimer-solvent density profiles at various temperatures. In Figs. 13(a) and $13(\mathrm{~b})$, we set $\epsilon_{D D}^{L J} / k_{b}=\epsilon_{D S}^{L J} / k_{b}=150 \mathrm{~K}$. It shows that the solvent profile does not change much at different temperatures except for $T=150 \mathrm{~K}$ at $\rho_{S} \sigma^{3}=0.1$, where there is apparent enhancement of density inside the dendrimer. The dendrimer generally shrinks as temperature decreases, while it is not so obvious for the $\rho_{S} \sigma^{3}=0.6$ case because of the excess solvent inside the dendrimer. In Figs. 14(a) and 14(b), we have $\epsilon_{D D}^{L J} / k_{b}=150 \mathrm{~K}, \epsilon_{D S}^{L J} / k_{b}=0$. For all temperatures but the athermal case, where all attractions vanish, the density of solvent inside the dendrimer is reduced greatly due to the unfavored interaction between the solvent and dendrimer. As temperature goes down, the separation between the dendrimer and solvent becomes more apparent and we see a highly collapsed dendrimer structure. There is semiquantitative agreement between iSAFT and simulation results except for the case of $\epsilon_{D D}^{L J} / k_{b}=\epsilon_{D S}^{L J} / k_{b}=150 \mathrm{~K}, T=150 \mathrm{~K}$ at $\rho_{S} \sigma^{3}=0.1$ [purple curve in Fig. 13(a)], which is equivalent to the case mentioned before.

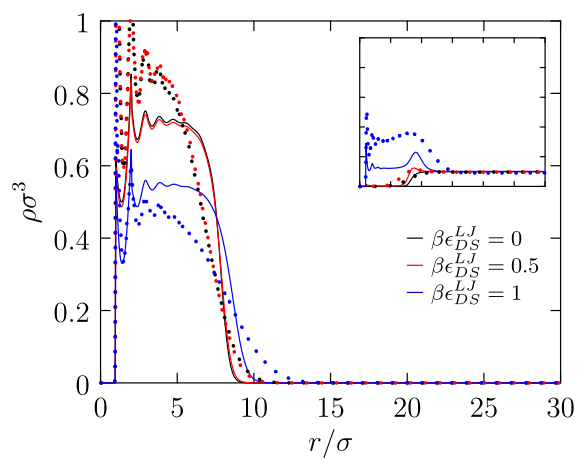

(a)

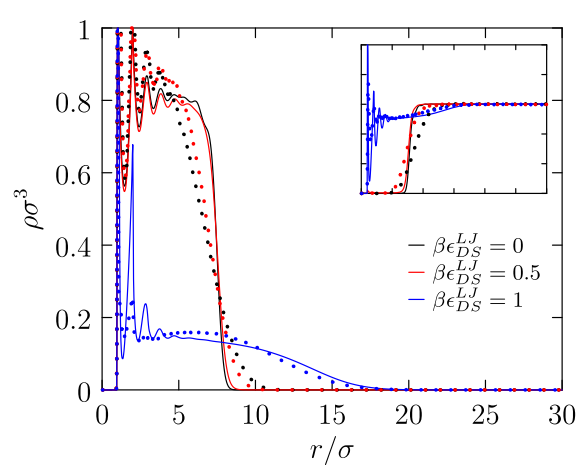

(b)
FIG. 12. Density profiles of $G 5$ dendrimer in spherical solvents of various affinity with dendrimer. $\beta \epsilon_{D D}^{L J}$ is fixed as 1. Solid lines represent iSAFT results, and dotted lines represent our simulation results. For clarity, density profiles of solvent segments are plotted in the inset with the same coordinate bounds and legends. (a) $\rho_{S} \sigma^{3}=0.1$ at bulk. (b) $\rho_{S} \sigma^{3}=0.6$ at bulk. 


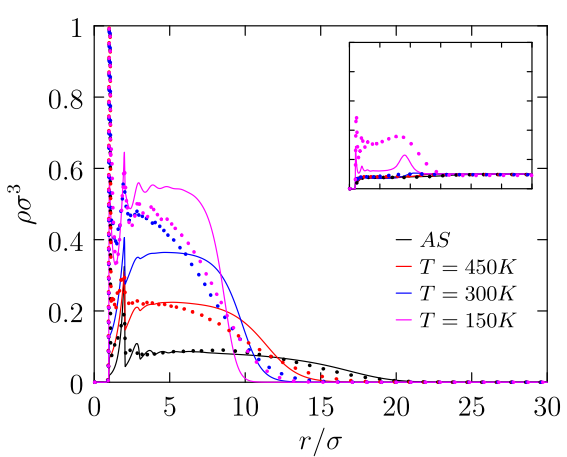

(a)

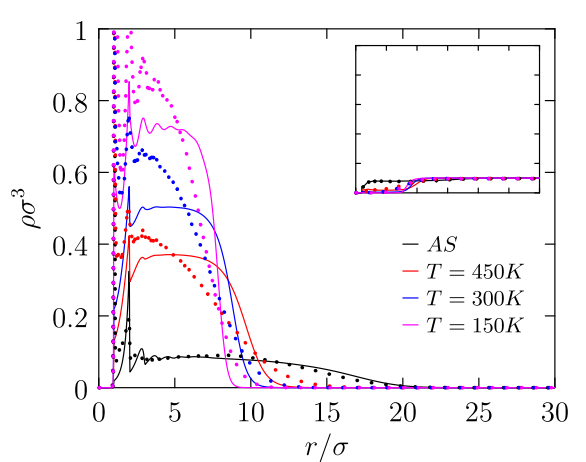

(a)

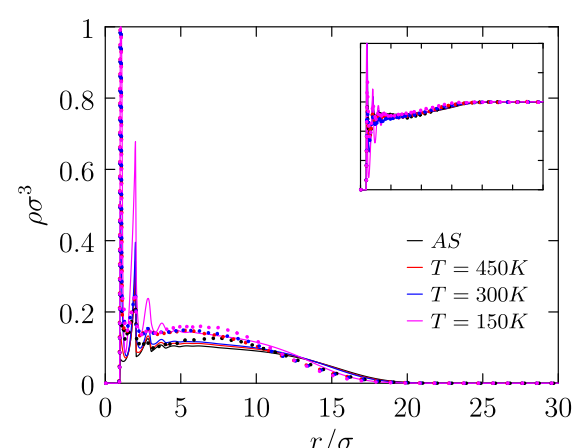

(b)

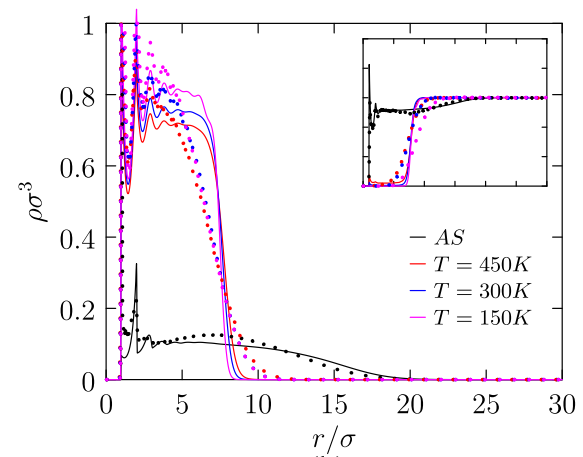

(b)
FIG. 13. Density profiles of $G 5$ dendrimer in spherical solvents at different temperatures. $\epsilon_{D D}^{L J} / k_{b}=\epsilon_{D S}^{L J} / k_{b}$ $=150 \mathrm{~K}$. Solid lines represent iSAFT results, and dotted lines represent our simulation results. For clarity, density profiles of solvent segments are plotted in the inset with the same coordinate bounds and legends. (a) $\rho_{S} \sigma^{3}=0.1$ at bulk. (b) $\rho_{S} \sigma^{3}=0.6$ at bulk.
FIG. 14. Density profiles of $G 5$ dendrimer in spherical solvents at different temperatures. $\epsilon_{D D}^{L J} / k_{b}=150 \mathrm{~K}$, $\epsilon_{D S}^{L J} / k_{b}=0$. Solid lines represent iSAFT results, and dotted lines represent our simulation results. For clarity, density profiles of solvent segments are plotted in the inset with the same coordinate bounds and legends. (a) $\rho_{S} \sigma^{3}=0.1$ at bulk. (b) $\rho_{S} \sigma^{3}=0.6$ at bulk.
It is concluded that for the simple model system considered here, a higher temperature always leads to a higher solubility regardless of the affinity between the dendrimer and solvent. However, this is not always the case in reality since real dendrimers have more interactions (polar, electrostatic, hydrogen bonding, etc.) other than repulsion and dispersion. Incorporating these complex interactions and extending to practical applications of dendrimer systems comprise the scope of our future work.

\section{CONCLUSION}

We have applied iSAFT to study the conformation of dendrimer molecules in both the implicit and explicit solvent. The density distribution of the dendrimer in an implicit solvent is in semi-quantitative agreement with the simulation data. The generation-by-generation density profile reveals that the details of the dendrimer and segments of outer generations are free to fold back to form a dense-core structure. When solvents are explicitly included, we have a large parameter space to explore that can affect the phase behavior of dendrimer. The solvent quality is affected by the solvent size, density, and chemical affinity with the dendrimer as well as temperature. Generally, solvents of smaller size and stronger interaction with the dendrimer tend to be candidates for good solvents. If only dispersion is considered, a higher temperature always improves the solvent quality. The iSAFT results of dendrimers in explicit solvents are well validated by our simulation results. Compared with molecular simulation, iSAFT has the great advantage of computational efficiency and the future work will include a comprehensive understanding of association on dendrimer systems and focus more on real applications, such as drug delivery.

\section{ACKNOWLEDGMENTS}

We thank the Robert A. Welch Foundation (Grant No. C-1241) for financial support.

\section{APPENDIX: SIMULATION DETAILS}

Molecular dynamics simulations were used to simulate a single dendrimer in implicit and explicit solvent conditions. The dendrimers were modeled as bead-spring freely jointed united-atom monomers. The solvent molecules were modeled as united atom spheres. The non-bonded interactions are calculated with a truncated and shifted Lennard-Jones potential, which is given as

$$
\begin{gathered}
u_{c s}^{L J}\left(r_{i j}\right)=u^{L J}\left(r_{12}\right)-u^{L J}\left(r_{c}\right), \\
u^{L J}\left(r_{12}\right)=4 \epsilon^{L J}\left[\left(\frac{\sigma}{r_{12}}\right)^{12}-\left(\frac{\sigma}{r_{12}}\right)^{6}\right],
\end{gathered}
$$

where $\epsilon^{L J}$ is the well depth, $\sigma$ is the particle diameter, and $r_{c}$ is the cutoff radius. The bonded monomer interactions is represented by a harmonic potential,

$$
u^{\text {bond }}\left(r_{12}\right)=K\left(r_{12}-r_{0}\right)^{2}
$$

where $K=1662 \epsilon^{L J} / \sigma^{2}$ is the spring constant ${ }^{60}$ and $r_{0}=\sigma$ is the equilibrium bond length. For all monomers and solvent particles, mass $m=1, \epsilon^{L J}=1, \sigma=1$, and $r_{c}=2.5 \sigma$.

We constructed the dendrimers with a series of selfavoiding random walks, using a modified version of the 
algorithm by Lescanec and Muthukumar. ${ }^{61}$ The modification was to ensure that the random walks were outwards from the center of the dendrimer. Hence, the dendrimer created is as open as possible, avoiding any configuration that can lead to monomers being trapped. In this study, we chose the branching factor as $b=3$ and the spacer length as $n=7$.

The simulations were carried using LAMMPS molecular dynamics code. ${ }^{62,63}$ The time step was chosen as $\Delta t=0.0006 \tau$, where $\tau=\sigma \sqrt{m / \epsilon^{L J}}$. The temperature $T^{*}=k_{B} T / \epsilon^{L J}$ was controlled by using the Nosé-Hoover thermostat with a damping factor of $3 \tau$. All simulations were minimized using a conjugate gradient method and initialized at a temperature of $T^{*}=6.5$. The system was then cooled to the desired temperature in $3 \times 10^{6}$ time steps. The system was further relaxed for $2 \times 10^{6}$ time steps. A larger time step and/or smaller damping factor can lead to better computational efficiency, which would reduce the equilibration time. The thermostat was then turned off and a production run was carried out for $10 \times 10^{6}$ steps, saving frames every 2000 time steps.

Implicit solvent simulations were carried out with one dendrimer in a vacuum. The reduced temperature changes the interactions between the monomers, hence acting like an effective solvent. Explicit solvent simulations were carried out by introducing solvent particles in random to the system. The dendrimer center was fixed at the origin of the cell, and periodic boundary conditions were established. The system size was set at $70 \sigma$, which ensures there is a minimum of $20 \sigma$ space between the dendrimer and its periodic image, avoiding any interactions between them. Solvent particles were inserted into the system in such a way that the total reduced density including the monomers and the solvents was the desired value.

To model hard sphere interactions, like the case of athermal solvent or no dendrimer-solvent interactions in explicit solvent cases, we changed the cutoff radius to $r_{c}=2^{1 / 6} \sigma$. It is important to note that those cases correspond to $\epsilon^{L J}=1$ with $r_{c}$ $=2^{1 / 6} \sigma$ in simulations, but labeled as $\epsilon^{L J}=0$ for uniformity. In addition, the temperature for athermal case was fixed ${ }^{23}$ at $T^{*}=1.2$.

${ }^{1}$ M. Fischer and F. Vögtle, Angew. Chem., Int. Ed. 38, 884 (1999).

${ }^{2}$ B. Klajnert and M. Bryszewska, Acta Biochim. Pol. 48, 199 (2001).

${ }^{3}$ M. Seiler, Chem. Eng. Technol. 25, 237 (2002).

${ }^{4}$ M. Wells and R. M. Crooks, J. Am. Chem. Soc. 118, 3988 (1996).

${ }^{5}$ F. Zeng and S. C. Zimmerman, Chem. Rev. 97, 1681 (1997).

${ }^{6}$ H. Tokuhisa, M. Zhao, L. A. Baker, V. T. Phan, D. L. Dermody, M. E. Garcia, R. F. Peez, R. M. Crooks, and T. M. Mayer, J. Am. Chem. Soc. 120, 4492 (1998).

${ }^{7}$ W. Li, T. Dohi, M. Hara, S. Nagano, O. Haba, K. Yonetake, and T. Seki, Macromolecules 45, 6618 (2012).

${ }^{8}$ J. F. Jansen, E. de Brabander-van den Berg, E. Meijer et al., Science 266, 1226 (1994).

${ }^{9}$ U. Boas and P. M. Heegaard, Chem. Soc. Rev. 33, 43 (2004).

${ }^{10}$ H. W. I. Peerlings and E. Meijer, Chem. - Eur. J. 3, 1563 (1997).

${ }^{11}$ D. Astruc and F. Chardac, Chem. Rev. 101, 2991 (2001).

${ }^{12}$ C. J. Hawker and J. M. J. Fréchet, "Comparison of linear, hyperbranched, and dendritic macromolecules," in Step-Growth Polymers for HighPerformance Materials (ACS Publications, 1996).

${ }^{13}$ J. M. Fréchet et al., Science 263, 1710 (1994).

${ }^{14}$ R. M. Crooks, M. Zhao, L. Sun, V. Chechik, and L. K. Yeung, Acc. Chem. Res. 34, 181 (2001).
${ }^{15}$ L. M. Kaminskas, V. M. McLeod, C. J. Porter, and B. J. Boyd, Mol. Pharmaceutics 9, 355 (2012).

${ }^{16}$ E. R. Gillies and J. M. Fréchet, Drug Discovery Today 10, 35 (2005).

${ }^{17}$ C. J. Hawker, K. L. Wooley, and J. M. Fréchet, J. Chem. Soc., Perkin Trans. 1, 1287 (1993).

${ }^{18}$ M. Liu, K. Kono, and J. M. Fréchet, J. Controlled Release 65, 121 (2000).

${ }^{19}$ S. Stechemesser and W. Eimer, Macromolecules 30, 2204 (1997).

${ }^{20}$ S. De Backer, Y. Prinzie, W. Verheijen, M. Smet, K. Desmedt, W. Dehaen, and F. De Schryver, J. Phys. Chem. A 102, 5451 (1998).

${ }^{21}$ A. Topp, B. J. Bauer, D. A. Tomalia, and E. J. Amis, Macromolecules 32, 7232 (1999).

${ }^{22}$ M. Chai, Y. Niu, W. J. Youngs, and P. L. Rinaldi, J. Am. Chem. Soc. 123, 4670 (2001).

${ }^{23}$ M. Murat and G. S. Grest, Macromolecules 29, 1278 (1996).

${ }^{24}$ Y.-J. Sheng, S. Jiang, and H.-K. Tsao, Macromolecules 35, 7865 (2002).

${ }^{25}$ G. Giupponi and D. Buzza, J. Chem. Phys. 120, 10290 (2004).

${ }^{26}$ R. Scherrenberg, B. Coussens, P. van Vliet, G. Edouard, J. Brackman, E. de Brabander, and K. Mortensen, Macromolecules 31, 456 (1998).

${ }^{27}$ E. G. Timoshenko, Y. A. Kuznetsov, and R. Connolly, J. Chem. Phys. 117, 9050 (2002).

${ }^{28}$ E. G. Timoshenko, Y. A. Kuznetsov, and G. E. Simonov, Physica A 379, 23 (2007).

${ }^{29}$ S. V. Lyulin, L. Evers, P. van der Schoot, A. A. Darinskii, A. V. Lyulin, and M. Michels, Macromolecules 37, 3049 (2004).

${ }^{30}$ M. Ballauff and C. N. Likos, Angew. Chem., Int. Ed. 43, 2998 (2004).

${ }^{31}$ C. Chen, P. Tang, F. Qiu, and A.-C. Shi, J. Phys. Chem. B 120, 5553 (2016).

${ }^{32}$ S. Jain, A. Dominik, and W. G. Chapman, J. Chem. Phys. 127, 244904 (2007).

${ }^{33}$ A. Bymaster and W. G. Chapman, J. Phys. Chem. B 114, 12298 (2010).

${ }^{34}$ R. Evans, Fundam. Inhomogeneous Fluids 1, 85 (1992).

${ }^{35}$ S. Tripathi and W. G. Chapman, J. Chem. Phys. 122, 094506 (2005).

${ }^{36}$ J. Wu, AIChE J. 52, 1169 (2006).

${ }^{37}$ C. P. Emborsky, Z. Feng, K. R. Cox, and W. G. Chapman, Fluid Phase Equilib. 306, 15 (2011).

${ }^{38}$ M. Wertheim, J. Stat. Phys. 35, 19 (1984).

${ }^{39} \mathrm{M}$. Wertheim, J. Stat. Phys. 35, 35 (1984).

${ }^{40}$ M. Wertheim, J. Stat. Phys. 42, 459 (1986).

${ }^{41}$ M. Wertheim, J. Stat. Phys. 42, 477 (1986).

${ }^{42}$ C. J. Segura, W. G. Chapman, and K. P. Shukla, Mol. Phys. 90, 759 (1997).

${ }^{43}$ Y.-X. Yu and J. Wu, J. Chem. Phys. 116, 7094 (2002).

${ }^{44}$ Y.-X. Yu and J. Wu, J. Chem. Phys. 117, 2368 (2002).

${ }^{45}$ S. Jain, "Molecular modeling of microstructure and thermodynamics of bulk and inhomogeneous polymer systems," Ph.D. thesis, Rice University, 2009.

${ }^{46}$ S. Jain, P. Jog, J. Weinhold, R. Srivastava, and W. G. Chapman, J. Chem. Phys. 128, 154910 (2008).

${ }^{47}$ K. Gong and W. G. Chapman, J. Chem. Phys. 135, 214901 (2011).

${ }^{48}$ K. Gong, B. D. Marshall, and W. G. Chapman, J. Chem. Phys. 137, 154904 (2012).

${ }^{49}$ K. Gong, B. D. Marshall, and W. G. Chapman, J. Chem. Phys. 139, 094904 (2013).

${ }^{50}$ A. Bymaster, S. Jain, and W. G. Chapman, J. Chem. Phys. 128, 164910 (2008).

${ }^{51}$ L. Wang, A. Haghmoradi, J. Liu, S. Xi, G. J. Hirasaki, C. A. Miller, and W. G. Chapman, J. Chem. Phys. 146, 124705 (2017).

${ }^{52}$ D. Chandler and J. D. Weeks, Phys. Rev. Lett. 25, 149 (1970).

${ }^{53}$ J. D. Weeks, D. Chandler, and H. C. Andersen, J. Chem. Phys. 54, 5237 (1971).

${ }^{54}$ Y. Rosenfeld, Phys. Rev. Lett. 63, 980 (1989).

${ }^{55}$ J.-P. Hansen and I. R. McDonald, Theory of Simple Liquids (Elsevier, 1990).

${ }^{56}$ W. G. Chapman, "Theory and simulation of associating liquid mixtures," Ph.D. thesis, Cornell University, 1988.

${ }^{57}$ S. Blokhina, N. Usol'Tseva, M. Ol'Khovich, and A. Sharapova, J. Anal. Chem. 62, 559 (2007).

${ }^{58}$ B. W. Koo, C. K. Song, and C. Kim, Sens. Actuators, B 77, 432 (2001).

${ }^{59}$ A. S. Kovvali, H. Chen, and K. K. Sirkar, J. Am. Chem. Soc. 122, 7594 (2000).

${ }^{60}$ N. W. Suek and M. H. Lamm, Macromolecules 39, 4247 (2006).

${ }^{61}$ R. L. Lescanec and M. Muthukumar, Macromolecules 23, 2280 (1990).

${ }^{62}$ S. Plimpton, J. Comput. Phys. 117, 1 (1995).

${ }^{63}$ Sandia National Laboratories, Lammps, http://lammps.sandia.gov, 2018. 